\title{
La trasformazione dell'impianto fortificato della città di Nola tra Quattrocento e Cinquecento
}

The transformation of the walls in the city of Nola between the fifteenth and sixteenth centuries

\author{
Giuseppe Mollo ${ }^{\text {a }}$, Giuseppe Piccolo ${ }^{\text {b }}$ \\ ${ }^{a}$ Dipartimento di Architettura - Università degli Studi di Napoli Federico II, Naples, Italy, gimollo@libero.it \\ b Independent, Nola, Italy, giuseppepiccolo@alice.it
}

\begin{abstract}
The study relating to the town walls of Nola (a city near Naples) is one of the most interesting among those about the Neapolitan military architecture between the fifteenth and sixteenth centuries. The building phases of the defensive walls allow us to retrace two main moments of the Renaissance military architecture.

It is to say, from the original and most ancient example of a puntoni struts fortifications, about which we can relate thanks to the precious and detailed Ambrogio Leone de Nola's description made at the beginning of 1500 , to the renewed defensive rampart body designed by el comendador Luis Escrivà during the Toledian period. A thorough recon of the iconographic sources, the regarding of the specialistic historiography, the contemporary theoretical assumptions and, most of all, a thorough recon of the still visible traces in the urban fabric and those emerged during the recent archeological investigations enable us to propose a structured theory about the building of the walls of Nola.
\end{abstract}

Keywords: Urban project, fortification, historical cartographies, Nola.

\section{Introduzione}

La contea di Nola, istituita da re Carlo I d'Angiò, fu concessa a Guido conte di Monfort nel 1269 e acquisita dagli Orsini nel 1290, in seguito al matrimonio di Anastasia, figlia di Guido, con Romano Orsini (1292-1327), nipote del cardinale Matteo Rosso, familiare di papa Niccolò III (Vincenti, 1997, pp. 19-22). È molto probabile che in quegli anni, sia stato dato avvio al riassetto di una originaria fortificazione della città -di cui non conosciamo l'estensione- che già nell'altomedioevo aveva subito una notevole contrazione rispetto all'ampiezza dell'antica città romana. Il nuovo polo di attrazione costituito dalla cattedrale ridefinì $i$ tracciati viari che in parte ricalcavano gli assetti agrari della prima età imperiale, realizzando un nuovo collegamento, via Cortefellana, con il complesso religioso.
Esso manteneva idealmente una relazione, attraverso l'antica porta "clausa" con il "Templum Augusti", l'edificio più rilevante del foro di età romana (Fig. 1). La città medievale era delimitata da un semplice recinto murario intervallato da frequenti torri a base quadrata e sul limite meridionale era innestata la cittadella strutturata sul modello del cassero quadrilatero di matrice normanno-federiciana, con mastio centrale e torri angolari, intervallate sui lati nord e sud da semitorri rettilinee. L'abitato, all'interno delle mura, era qualificato dalla preminente funzione religiosa, costituita dal monumentale centro episcopale e dall' organizzata suddivisione degli spazi. Questi aspetti avevano determinato l'organizzazione complessiva in quartieri della città, come documentano le fonti della seconda 
metà del XIV secolo e dalle quali possiamo dedurre la presenza di un definito assetto urbano e recinto fortificato (Buonaguro, 1997, p.112).

$\mathrm{Nel}$ periodo angioino, la civitas indicata con l'appellativo di castrum Nole, la ritroviamo, con frequenza, associata al castello di Cicala situato sulla sommità dell'omonima collina nei pressi della città.

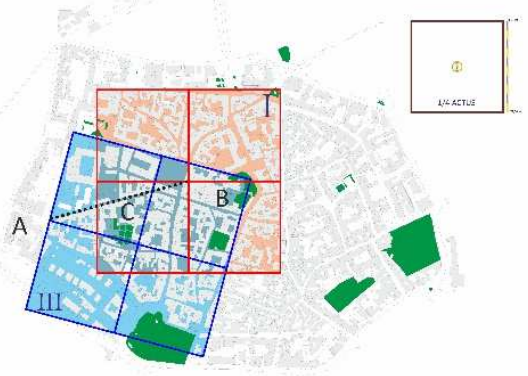

Fig. 1. Relazioni urbane tra la città antica e quella medievale. Assetti agrari tra la tarda età repubblicana Nola I (130-82 a.C) e prima età imperiale Nola III (6979 d.C.); A) Tempio di Augusto, B) Insula Episcopalis; C) Via Cortefellana.

\section{Nola, contea della famiglia Orsini}

Con il passaggio di Nola alla famiglia Orsini, il capoluogo della contea crebbe di importanza. $\mathrm{Si}$ allestì, in città, un grande cantiere di trasformazione di cui sono testimonianza soprattutto le architetture religiose: i monasteri francescani di S. Chiara voluti dal conte Roberto Orsini (13271345), quello di San Francesco ed il Collegio delle Vergini dal conte Nicolò Orsini (13451399). In questa prospettiva di continuo cambiamento, durante l'assedio che la città subì per diciotto mesi dal Re Ladislao di Durazzo, al tempo del conte Pirro Orsini (1399-1412), la cinta fortificata era efficiente e solida, ed è verosimile ipotizzare che, alla fine del Trecento, si apportarono modifiche significative alla cittadella che proteggeva l'ingresso principale alla città sul lato meridionale della fortificazione (Di Cerbo, 2013, pp. 22-28).

Lo stesso Ambrogio Leone, nel De Nola, si esprime in maniera dubitativa: "Arcem alii ad Ladislaum regem alii ad Pyrrhum Ursinum Nicolae filium referunt" e continuando "Raimundus Ursinus Pyrrhi filius promurale cum erismatibus fecit; idem semiturrem, quem umbonem vocavimus, ante hostium arcis externum construxit [...]" (Leone, 1514, Ca. xvi, c. xxxxr). Da queste affermazioni fu Raimondo Orsini (1415-1459), elevato dalla regina Giovanna II alla suprema carica di Grande Giustiziere del Regno, ad intraprendere queste opere di rinnovamento, grazie anche alle concessioni di re Alfonso che lo remunerò non solo con il principato di Salerno, "ma per obbligarselo vieppiù, gli diede nel 1437 per moglie Eleonora d'Aragona, figliola del conte di Aveglia o Argel, sua cugina e per dote il ducato d'Amalfi" divenendo così uno dei più ricchi signori della sua epoca (Vincenti, 1997, p. 29).

La definitiva conquista del Regno di Napoli da parte di Alfonso I (1442-1458) segnò l'avvio di un grande fervore costruttivo nell' area napoletana. Nel disegno complessivo del re, l'intervento sul territorio aveva carattere prioritario; in tale programma assunse particolare rilievo la realizzazione di efficienti reti viarie ed un efficace sistema difensivo. È ragionevole ipotizzare che le opere di perfezionamento delle fortificazioni nolane possano essere state avviate nel primo decennio della seconda metà del XV secolo e completate durante il periodo del regno di Ferrante I (1458-1494) suo successore.

Sono gli anni in cui si lavora alacremente nel cantiere di Castelnuovo e i maestri catalani, lì attivi, trovarono altre occasioni di lavoro nel territorio a nord della capitale, in centri come Capua, Carinola, Sessa Aurunca, Nola e Fondi, dove si registra una certa diffusione delle forme artistiche provenienti dalla penisola iberica. Ma i documenti napoletani, con la pausa delle guerre di successione dinastica (1458-1469), citano maestri catalani fino al 1480, a dimostrazione di un fecondo rapporto tra $\mathrm{i}$ due territori della Corona d'Aragona.

\subsection{Orso Orsini, dalla pergamena aragonese alle influenze albertiane}

Il successore di Raimondo fu Orso Orsini (14621479), figlio illegittimo di Gentile Orsini di Pitigliano. Nel 1462, aveva ottenuto in feudo Nola non come una legittima eredità di famiglia, bensì come ricompensa per il suo improvviso passaggio dalla parte angioina a quella aragonese durante la guerra di successione seguita alla morte del re Alfonso I d'Aragona, tra l'erede al trono Ferrante e il pretendente francese Giovanni d'Angiò (1458-1465). All'epoca Orso era già affermato e conosciuto in tutta la Penisola per le sue qualità militari. Al centro della trattativa per 
il passaggio dalla parte del re Ferrante era stata proprio Nola: la città fu tolta a Felice Orsini, esponente del ramo degli Orsini di Nola e dunque legittimo erede della contea nolana, per darla ad Orso.

L'evento ebbe notevole eco tra i contemporanei, e nel libro VII dei Commentarii Enea Silvio Piccolomini non esitò a descrivere il passaggio dell'Orsini alla parte aragonese come l'evento determinante per la risoluzione della guerra, conclusasi con la vittoria delle truppe di Ferrante nella battaglia di Troia del 18 agosto 1462 . Nel De bello Neapolitano l'umanista Giovanni Pontano fornisce l'eccezionale descrizione del rituale cavalleresco del ligio omagio attraverso cui Orso giurò fedeltà a Ferrante e a testimonianza del loro stretto rapporto, Pontano celebra Orso Orsini per le sue doti militari, per l'ingegno, per i gesti di liberalità verso i cittadini nolani e per la magnificenza del suo palazzo di Nola dedicandogli l'epigramma funerario in seguito alla sua morte avvenuta il 5 luglio 1479 a Viterbo, durante la guerra di Toscana (de Divitiis, 2016, p. 28).

La cultura di Orso e i suoi vasti interessi umanistici emergono dall'inventario della sua biblioteca napoletana. Egli stesso, inoltre, nel 1477 aveva composto un trattato militare in volgare, il Governo et exercitio de la militia, dedicato al re Ferrante. Un testo all'avanguardia per le nuove tecniche belliche e al contempo intriso di cultura classica, nel quale si illustra l'uso delle artiglierie leggere e viene proposto un progetto moderno di organizzazione militare.

Si potrebbe attribuire agli anni settanta del Quattrocento, la campagna di ricognizione e il progetto di rilevamento del territorio confluita nel disegno della pergamena conservata all'Archivio di Stato di Napoli (Valerio, 2015, pp. 208-211).

Nola è qui rappresentata come un ampio nucleo urbano completamente cinto da mura, sul quale si apre la porta principale alla cittadella posta a difesa dall'ingresso di porta Vicanzio.

$\mathrm{La}$ fortificazione appare ancora priva dell'antemurale e del rivellino che difende l'ingresso alla citta da sud, documentando quindi una situazione precedente alla riorganizzazione delle difese e dunque non priva di interessanti considerazioni (Fig. 2).

Pur senza riscontri, l'ipotesi più accreditata è che le mappe siano state disegnate forse dallo stesso
Gioviano Pontano agli inizi degli anni novanta del Quattrocento, tuttavia con molte perplessità, da parte degli studiosi, non può essere esclusa né la sua indiretta partecipazione alla stesura delle stesse e nemmeno una datazione anticipata.

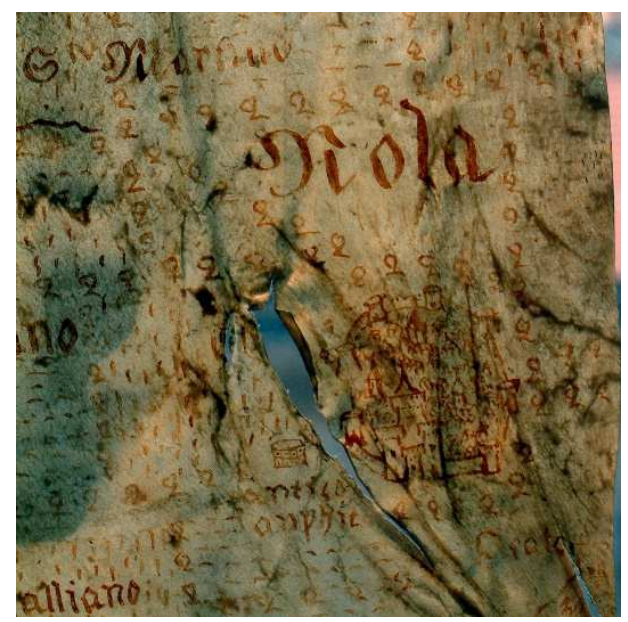

Fig. 2. Mappa del territorio tra Maddaloni, Acerra e Nola (prima metà del secolo XVI). Inchiostro carminio su pergamena; $32 \times 29 \mathrm{~cm}$ ca. (Napoli, Archivio di Stato, Archivio farnesiano, Piante e disegni, n. 65).

Coincidenze, o meno, siamo nella fase, del coevo cantiere di Castelnuovo, caratterizzata dall'incontro di maestranze catalane con scalpellini di educazione rinascimentale toscana, iniziata nel 1465 , lo stesso anno di una documentata visita di Leon Battista Alberti a Napoli, compiuta forse per sfuggire ad una pestilenza che aveva colpito Roma (Borsi, 2006, p. 345). Condividiamo l'ipotesi avanzata dagli studiosi circa la diretta partecipazione del conte Orso all'ideazione dell'ampliamento della cinta muraria nolana, che ripercorre "interessanti canali di trasmissione di modelli culturali e artistici, che giustificano la presenza di soluzioni influenzate dal lessico introdotto dalla scuola albertiana in tutta la produzione rinascimentale napoletana" (Caianello, 2003, pp. 30-31).

\subsection{Nola Praesens}

Così Ambrogio Leone nella prefazione al $D e$ Nola: "Io con l'aiuto del pittore Girolamo Moceto l'ho raffigurata e disegnata con tanta somiglianza da poter esser assai facilmente visibile agli occhi di tutti e in ogni parte della terra [...] Ho deciso, infatti, di onorare la patria in questa 
operetta con la fedele immagine e con la pura storia. La forza, difatti, e l'azione dell'immagine è veramente divina, poiché rende ed offre l'aspetto vero e proprio di ogni cosa e con tanta esattezza la mostra presente anche se assente, come se fosse sotto i propri occhi" (Leone, 1514, c. IIV).

La rappresentazione urbana della città di Nola agli inizi del XVI secolo incisa da Girolamo Mocetto nel De Nola di Ambrogio Leone descrive con efficacia la città dei suoi tempi e il suo apparato difensivo: l'addizione alla cinta muraria più antica con un singolare e innovativo sistema costituito dall'antemurale promurali e dal contrafforte verbo novo barbacanis su cui si innestano, ad intervalli regolari, le torri a base triangolare. Il disegno accurato anche nei dettagli è stato realizzato a partire da piante e prospetti o schizzi misurati da cui è stata poi realizzata la veduta prospettica da sud (Hind, 1948, V, pp. 170-171, VII, pp. 734-735) (Fig. 3).

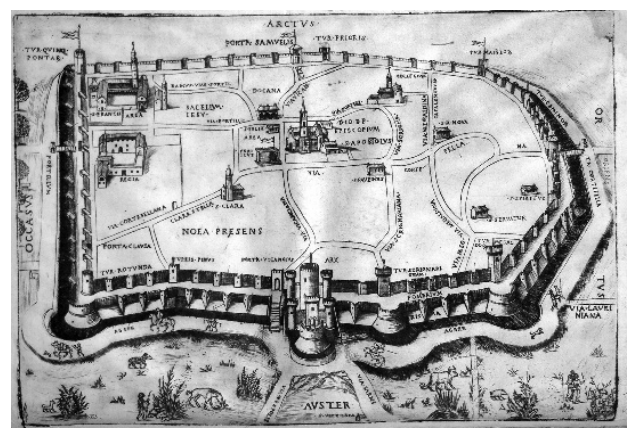

Fig. 3. Ambrogio Leone, Girolamo Mocetto, Nola Praesens, inizi XVI secolo, incisione su rame; De Nola opusculum [...], Venezia, 1514.

All'interno degli isolati urbani -separati dalle strade identificate con i loro nomi- emergono in alzato gli edifici più significativi: la cattedrale, le chiese, l'episcopio, i monasteri, la dogana, il seggio e il palazzo del conte (Regia). Una simile rappresentazione trova riscontro nell'iconografia urbana medievale di Venezia, nota nella copia realizzata da Cristoforo Sabbadino del 1557 (de Divitiis, 2018, p. 81-102). La pianta allegata al testo cinquecentesco del De Nola come evidenziato dagli studi si inserisce tra le prime rappresentazioni iconografiche e narrazioni di centri urbani, anticipatrice dei futuri "libri delle città" (Di Cerbo, 2013). Girolamo Mocetto ha inciso le lastre e assieme al Leone ha giocato un ruolo decisivo nella definizione e nella scelta dei dettagli. Disegni che il Leone in forma di schizzi e appunti aveva portato con se da Nola assieme ad appunti e informazioni di carattere storico. Un lavoro a due mani fatto di schemi e concetti che il Mocetto declina con la sua arte di pictor.

L'autore del De Nola delinea una configurazione urbana scandita dalle quattro porte principali ciascuna collocata in corrispondenza dei punti cardinali e dalle torri, tutte posizionate nei punti dove la cortina muraria si piega ad angolo. Poi immaginando una sezione ideale e fornendo anche le misure in piedi indica nel pomerium lo spazio libero da costruzioni che corre parallelo all'interno del recinto murario, analogamente, ma meno largo di quello esterno, che separava il muro di cinta dal promurale, quest'ultimo concluso da un merlatura. Sul contrafforte (erisma) sono costruite delle torri triangolari a forma di piramidi capovolte (trilatere metae inversae) e negli spigoli, sul lato della cortina del Portellum rispettivamente la Turris Rotunda posta nell'angolo sud-ovest e la Turris Quinque pontarum situata nell'angolo nord-ovest; infine un ampio fossato e un terrapieno su cui corre la via circondano la città (Fig. 4). Sul versante meridionale era la cittadella (Arcem/Arx), imponente costruzione a pianta quadrata, dotata di spesse mura, con quattro torri minori negli angoli ed una maggiore al centro alta 120 piedi, circondata da un fossato e con la porta principale difesa da un rivellino a forma di mezzaluna (est in speciem hemicycli figurata, cuius curvatura et umbo exterior est) (Leone, 1514, Ca viii, c.xxixr). I riferimenti teorici, come abbiamo evidenziato, sono quelli da ricercare nel De re aedificatoria di Leon Battista Alberti che trattando dei diversi modi di fortificare la città, consiglia, il doppio muro: "Interposto uno spatio di venti piedi, faccinvisi duoi muri dallo lato di dentro, \& infra loro vi si getti la terra che si cava de fossi, [...] Faccinsi Barbacani fuori del diritto delle mura, a guisa di Triangolo, con uno angolo volto a nimici, discosto l'uno da l'altro sette braccia \& mezo [...]" (Alberti, 1565, libro IV, cap. IIII, p. 81). 
Rimane, a nostro avviso, significativa l'ipotesi avanzata dal Promis, alla fine dell'Ottocento, che tra numerosi esempi collocava il barbacane di Nola tra quelli più originali ed innovativi datandolo alla prima metà del XV secolo. Lo stesso autore si soffermerà anche su altri due aspetti della fortezza nolana: lo spalto, che in epoca tarda sarà modellato a scarpa verso la campagna e il rivellino a pianta semicircolare situato sul fronte dell'arce, su cui proporrà stimolanti confronti (Promis, 1841, pp. 204, 273, 218-219).

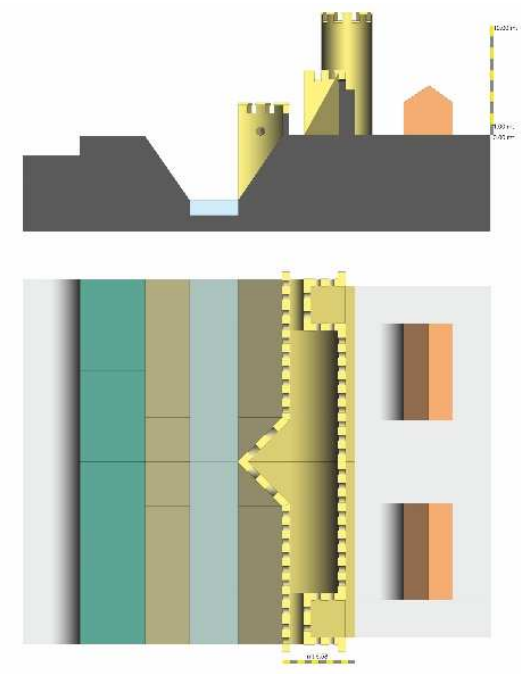

Fig. 4. Ricostruzione in pianta e sezione della cinta muraria "leonina" (Elaborazione grafica arch. G. Piccolo).

\section{Nola nel viceregno: un cantiere infinito}

A seguito dei privilegi concessi da Carlo V e per l'importanza che Nola ricopriva nel contesto territoriale, in funzione dello status di città appartenente al Regio Demanio, cominciò a delinearsi la necessità di costruire una nuova fortificazione, in grado di assolvere alle esigenze legate alla mutata tecnica bellica e che contribuisse a dare un'immagine di potenza e di prestigio, così come stava avvenendo per altre città del Regno, come Capua e L'Aquila.

Le parti del nuovo sistema di fortificazione dovevano essere concepite come un complesso unitario in cui forma e dimensioni di ciascun elemento dovevano essere in preciso rapporto geometrico con tutte le altre componenti, pena la vulnerabilità dell'intero complesso. A queste nuove esigenze s'ispirerà il progetto affidato dal vicerè di Napoli Don Pedro di Toledo al cavallero valenciano Pedro Luis Escrivà, già attivo a Napoli durante l'assedio dei francesi del 1528, e assunto nel 1532 per dirigere i lavori dei cantieri napoletani e in Terra di Lavoro. Nel corso di un sopralluogo del vicerè, alle fortificazioni capuane, dovettero, tuttavia, emergere la scarsa efficacia di alcune soluzioni proposte dal Commendador Escrivá. Il vicerè commissionò, dunque, nuovi disegni e la fabbrica capuana proseguì con lentezza fino all'intervento del regio architetto Giangiacomo dell'Acaya nel 1542 (Brunetti, 2006b, p. 37).

In particolare, come egli stesso ricorda nella Apologia, nel rispondere alle critiche che gli erano state mosse sulle scelte progettuali del castello di Sant'Elmo, trattando delle facce dei baluardi coperte da una sola troniera, spiegava come dovessero essere piegate le difese in quel punto chiarendo quale avrebbe dovuto essere l'inclinazione dei baluardi rispetto alla cortina (Escrivá, 2000, p. 104).

Per risolvere il problema, l'Escrivà richiamava i progetti di Nola e Capua, dove aveva disegnato una cortina articulada sul modello di un fronte bastionato concepito dal duca Alfonso I d'Este per la fortificazione della città di Ferrara e che ricorda il baluardo a fianchi doppi Ardeatino eretto nel 1534 su progetto di Antonio da Sangallo il giovane. La soluzione proposta dall'Escrivá non fu mai realizzata, al loro posto si costruirono semplici cortine, ascrivibili ad una fase progettuale affidata ad altri professionisti. Smantellate le mura quattrocentesche fu deciso di recingere la città con una serie di cortine rettilinee costituenti un poligono di sette lati con altrettanti baluardi pentagonali, innestati nei vertici e modellati per inglobare le aree di sedime delle vecchie torri. L'antico castello, invece, fu risparmiato dalla distruzione e, mentre nella fortificazione rinascimentale era parte della linea 
esterna di difesa, nella nuova sistemazione fu ricompreso nell'area del nuovo recinto bastionato (Fig. 5). E così fino al 1886, quando fu demolito per dubbie esigenze di sanità pubblica (Carillo, 1993, pp. 380-401; Mollo, 2015, pp. 2532).

La scelta di fortifcare alla moderna una città dalle dimensioni come Nola non poteva essere priva di conseguenze. Tralasciando i disagi e i danni arrecati alle popolazione per le complesse opere preparatorie di demolizione delle mura quattrocentesche e il livellamento del terreno, la gestione e la reale capacità di tenuta di una fortezza, richiedeva inevitabilmente l'impiego di risorse economiche e umane tali da mettere in difficoltà la città.

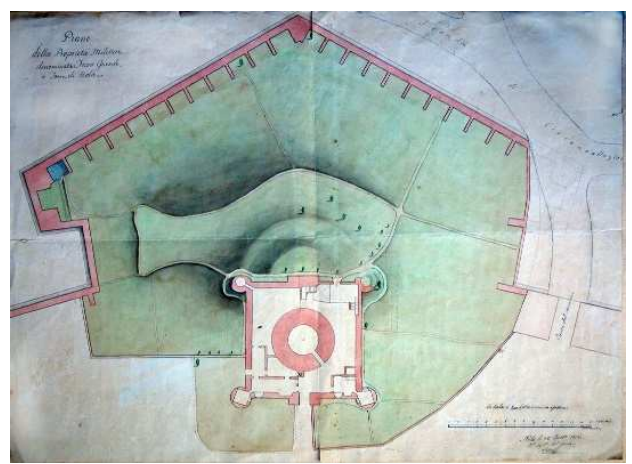

Fig. 5. Fosso Grande o Torre di Nola, 28 febbraio 1816 (Napoli, Biblioteca Nazionale Vittorio Emanuele, Fondo manoscritti e rari, b.5C95).

Le fonti comprovano questi impedimenti già a partire dal 24 gennaio 1541. Nel corso della visita del vicerè si rilevava la mancanza di economie per ultimare questo come altri cantieri e nel resoconto inviato a Francesco de Los Cobos, sui margini dei fogli furono annotate le somme necessarie al loro completamento (AGS fascio 1024/42; Mauro, 1998, p.255). E ancora, il 25 giugno 1573 con l'aggiunta di controlli al fine di evitare "frodi nella costruzione della fabbrica" e il 22 marzo del 1596 quando si rese necessaria l'imposizione di altre gabelle ai cittadini, come riportato nell'Istrumento della convenzione tra l' Università di Nola e la Regia Corte (Caianello, 2003, p. 48, n. 86; Brunetti, 2006a, p. 40).

\section{La fortificazione disegnata}

L'interesse per la fortificazione della città di Nola è confermata anche da sette disegni: i primi due fanno parte di un gruppo di disegni raccolti in due album conservati presso la Biblioteca dell'Istituto Storico di Cultura dell'Arma del Genio di Roma.

Il primo disegno, incluso nella raccolta più antica, dal titolo Forti di Uropa, datata al 1590, è stato attribuito a Tiburzio Spannocchi (15411606), che avrebbe disegnato numerosi centri del Regno di Napoli a partire dal 1573; il secondo disegno, contenuto nel secondo album è stato invece attribuito a Matteo Neroni, si tratta di un disegno a penna colorato, orientato a nord-est, nel quale si evidenzia la fascia di rispetto interna ai baluardi, individuati in legenda con la loro rispettiva denominazione: A Gonzaga, B Vittoria, C Alba, D (senza nome), E Reale, F Castello, G Felice, H Porta, (Fig. 6). Lo schema di base di questo disegno si confronta con quello ad inchiostro bruno, conservato nel Gabinetto delle Stampe degli Uffizi di Firenze (GDSU f. 4258A); sempre a Firenze, nella Biblioteca Nazionale, è il disegno a penna acquerellato realizzato agli inizi del Seicento per un "atlante di presentazione" attribuito anch'esso a Matteo Neroni (BNCF, Mscr. II.I.281.41) (Caianello, 2003, pp. 35-41; Brunetti, 2006a, pp. 40-41).

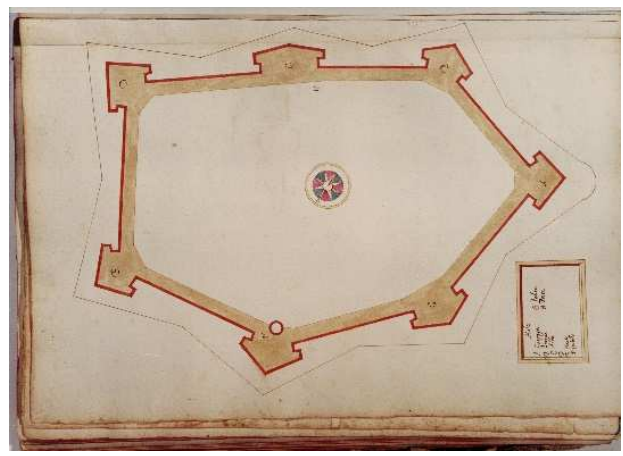

Fig. 6. Matteo Neroni (attr.), Nola, inizi XVII secolo, BB951/C51.140 (Roma, Biblioteca dell' Istituto Storico di Cultura dell' Arma del Genio).

A questi vanno aggiunti altri tre schemi che descrivono due diversi progetti fortificazione della città. I primi due sono conservati nella 
biblioteca nazionale di Francia ed illustrano l'ampliamento della fortificazione sul lato settentrionale con l'aggiunta di due baluardi con due differenti proposte di innesto delle nuove cortine ai baluardi esistenti (BNF, Royaume des deux Siciles, IV, Pr. Terre de Labour, P60562; Brunetti, 2006a, pp.38-39). Il terzo disegno di progetto è quello della collezione le Poivre nella Biblioteca del Palacio Real di Madrid. La planimetria rappresentata è analoga al secondo disegno riportato sul foglio di Parigi, particolare è il dettaglio della doppia cortina di connessione alla cinta preesistente e l'aggiunta di orecchioni sui fianchi ritirati del baluardo "Gonzaga" (BPRM, Designo de Nola y sus Relaçiones, Pierre le Poivre, MAP/416.8; Mollo, 2016, pp. 149-154).

Senza peraltro voler attribuire la paternità di questi disegni all'uno all'altro degli ingegneri regi o esperti che si sono alternati nel corso della costruzione della fabbrica nolana è opportuno segnalare come le fonti attestino nel febbraio del 1557 l'incarico commissionato dal duca d'Alba a Vespasiano Gonzaga per "lo designo de dicta fortificatione"; nel gennaio del 1565 a Luigi
Boggiolo collaboratore di Giovanni Tommaso Scala "per il disegno fatto della fortezza" ed in una nota del notaio Nicola Simone, nel febbraio del 1582, a Benvenuto Tortelli "ingegnero deputato alla fortificatione di Nola per lo novo disegno fatto" (Caianello, 2003; Mollo 2016).

Sulla scorta della documentazione d'archivio e grazie ai ritrovamenti di brani della fortezza vicereale (Fig. 7) si propone in questa sede un disegno che restituisce in un unica tavola i due recinti murari (Fig. 8).

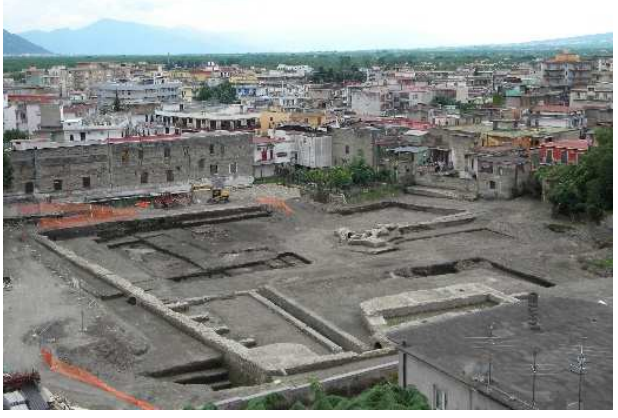

Fig. 7. Il baluardo "Vespasiano Gonzaga", il cantiere archeologico 20/05/2010 (Nicola Castaldo).

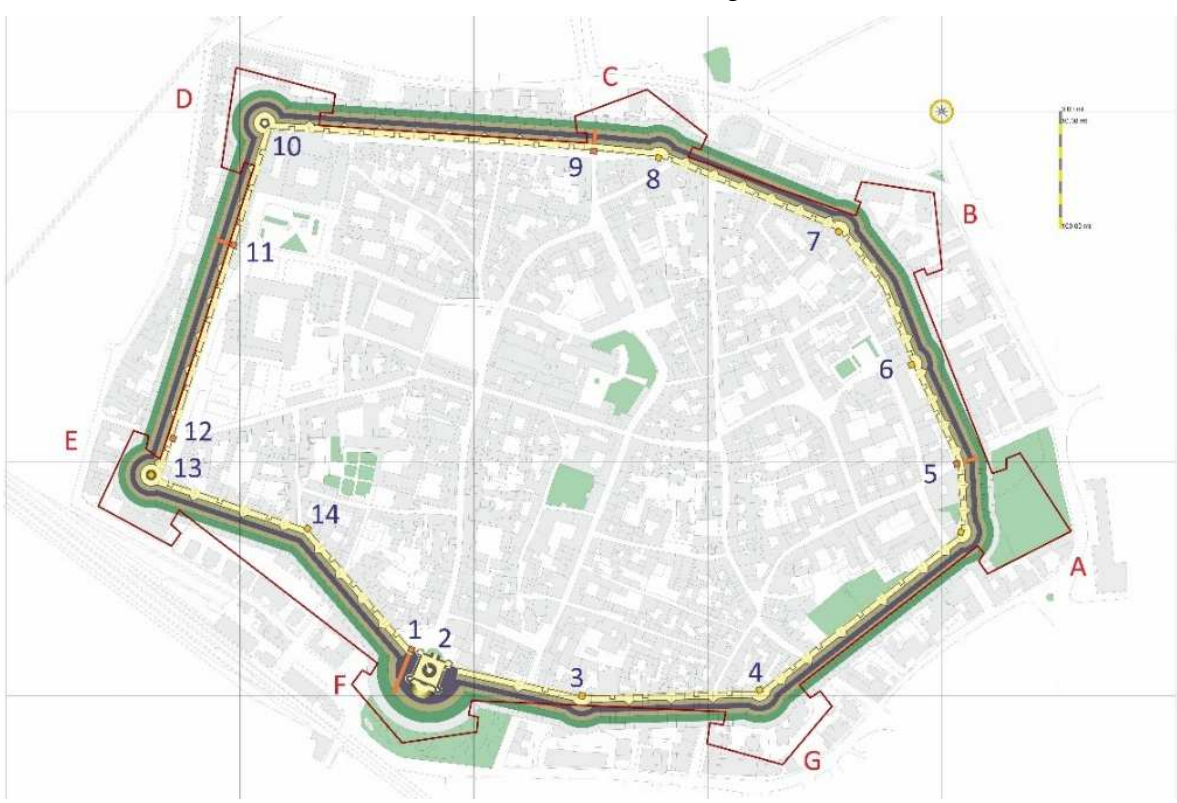

Fig. 8. Ipotesi ricostruttiva delle fortificazioni. Rinascimentale, torri e porte: 1.Porta Vicanzio, 2.Arce, 3.Torre degli Scrignario, 4.Torre dei Megaldo, 5.Torre/Porta Cortefella, 6.Torre dei Cimino, 7.Torre dei Mazzeo, 8.Torre del Priore, 9.Torre/Porta di Samuele, 10.Torre "quinque pontar", 11.Portellum, 12.Porta "clausa", 13.Torre Rotonda,14.Torre del Pino. Vicereale, baluardi: A.Gonzaga, B.Vittoria, C.Alba, D. (s.n.), E. Reale, F. Castello, G. Felice. (Restituzione grafica su base aerofotogrammetrica scala 1:1000. arch. G. Piccolo). 


\section{Conclusioni}

La complessità tecnica ed economica nella costruzione di una fortezza come quella di Nola, che andava sperimentando soluzioni innovative, necessitava di un continuo aggiornamento nella progettazione, questo comportò, un aumento delle spese e il conseguente rallentamento dei lavori, che non furono più al passo dei tempi, come risulta nella relazione dell'ambasciatore Veneto Giovanni Ramusio residente a Napoli tra il 1594 ed il 1597 che definisce "impefetta" la fabbrica nolana (Caianello, 2003, p.48) e nella cronaca del Governatore di Lauro Giovan Battista Piacente che così descrisse la città nella prima metà del XVII secolo: "Nola [...] è situata in piano, e cinta da una fortissima muraglia alla moderna, ma per l'esorbitanza della spesa non ancora all'ultima perfezione ridotta: è fiancheggiata da sette vastissimi baluardi, dalla sommità dei quali non è parte del recinto, che non resti scoperta: finalmente gode tutti quei requisiti, che per una lunga e gagliarda difesa si potrebbero ricercare" (Piacente, 1861, p. 163).

\section{Bibliography}

Alberti, L.B. (1565). L'architettura di Leon Battista Alberti tradotta in lingua fiorentina da Cosimo Bartoli Gentil'huomo e Accademico fiorentino, Monte Regale appresso Lionardo Torrentino.

Borsi, S. (2006). Leon Battista Alberti e Napoli, Firenze.

Brunetti O. (2006a). L'ingegno delle mura. L'Atlante Lemos della Bibliothèque Nationale de France, Firenze, pp. 38-41.

Brunetti. (2006b). A difesa dell'Impero. Pratica architettonica e dibattito teorico nel Viceregno di Napoli nel Cinquecento, Lavello.

Buonaguro, C. (1997). Documenti per la storia di Nola secoli XII-XIV, Nocera Inferiore.

Caianiello, M.C. (2003). "La cinta fortificata di Nola tra Quattrocento e Cinquecento", Castellum, 45, pp. 27-50.

Carillo, S. (1993). "La distruzione della torre medievale di Nola", in Fiengo G. ed., Tutela e restauro dei monumenti in Campania 1860-1900, Napoli, pp. 380-401.

Cerbo, C. Di. (2013). "La Nola degli Orsini tra XIII e XIV secolo: topografia, sistema difensivo, castrum e magnificazione della città”, Archivio Storico per le Province Napoletane, XXXI, pp. 1-28.

Divitiis, B. de. (2016). "Rinascimento meridionale: la Nola di Orso Orsini tra ricerca dell'antico e nuove committenze", Annali di Architettura, 28, pp. 27-48.

Divitiis, B. de. (2018). "Architecture and Nobility: The Descriptions of Buildings in the De Nola" in de Divitiis, B.; Lenzo, F.; Miletti, L. eds., Ambrogio Leone's De Nola, Venice 1514 Humanism and Antiquarian Culture in Renaissance Southern Italy, Leiden-Boston, pp. 81-102.

Escrivá, P.L. (2000). Apologia en escusation y favor de las fabricas del Reyno de Napoles del Commendador Scriva, in Sanchez-Gijón, A.; Cobos Guerra, F.; Castro Fernandez, J. De. transc., Luis Escrivá su apologia y la fortificación imperial, Generalitat Valenciana, Valencia, pp. 38-181.

Hind, A.M. (1948). Early Italian Engraving. A Critical Catalogue with Complete Reproduction of All the Prints Descrived, London.

Leone, A. (1514). De Nola opusculum distinctum, plenum, clarum, doctum, pulcrum [sic], verum, grave, varium, et utile, Giovanni Rosso Vercellano, Venezia.

Mauro, A. (1998). Le fortificazioni nel Regno di Napoli, Napoli.

Mollo, G. (2015). "La distruzione della torre medievale di Nola in un inedito documento del 1886", Agorà del Vallo di Lauro, 19, Domicella, pp. 23-30.

Mollo, G. (2016). "L'ampliamento della cinta fortificata vicereale di Nola nei disegni dell' Atlante Lemos della Bibliothèque Nationale de France e nella collezione di Pierre le Poivre della Biblioteca Real di Madrid", in Verdiani, G. ed., Defensive architecture to mediterranean coast XV to XVII centuries, vol. III, Firenze, pp. 149-154.

Piacente, G.B. (1861). Le rivoluzioni del Regno di Napoli negli anni 1647-48 e l'assedio di Piombino e Portolongone, Napoli.

Promis, C. (1841). Dello stato dell'Architettura Militare circa l'anno millecinquecento e dell'origine delle singole parti della fortificazione conosciute a quell'epoca, III, in: Trattato di Architettura civile e militare di Francesco di Giorgio Martini [...] per cura del cavaliere Cesare Saluzzo, Torino, pp. 201-281.

Valerio, V. (2015). "La cartografia rinascimentale del regno di Napoli. Dubbi e certezze sulle pergamene geografiche aragonesi”, Humanistica an International Journal of Early Renaissance Study, X, pp. 191-232.

Vincenti, G. (1997). La contea di Nola dal sec. XIII al XVI, in Podda, M. di. ed., Comitato del Giglio della Corporazione dei Calzolai, ristampa dell'edizione del 1897, Nola. 